\title{
A RECIPE TO OBTAIN LIDAR POLARISATION CALIBRATION PARAMETERS G, H AND K
}

\author{
Joelle Buxmann ${ }^{1 *}$, Martin Osborne ${ }^{1}$, George Georgoussis ${ }^{2}$ and Volker Freudenthaler ${ }^{3}$ \\ ${ }^{1}$ Met Office, FitzRoy Road, Exeter, Devon, EX1 3PB, United Kingdom \\ ${ }^{2}$ Raymetrics S.A., Spartis 32, Metamorfosis, Greece. \\ ${ }^{3}$ Ludwig-Maximilians-University, Munich, Germany \\ *Email: Joelle.c.buxmann@metoffice.gov.uk
}

\begin{abstract}
The accuracy of the polarisation calibration is of prime importance for aerosol classification using lidars. We present a detailed description how to obtain the calibration parameters introduced in 2016 [1] accounting for various effects of non-ideal optics, lasers and atmospheric conditions. We find that crucial parameters such as the rotation angle of the plane of polarisation of the Laser $(\operatorname{Rot} L)$ as well as the degree of linear polarisation $(D O L P)$ influence the volume linear depolarisation ratio significantly.
\end{abstract}

\section{INTRODUCTION}

In a paper on the effects of polarising optics on lidar signals and the polarisation calibration [1] a set of general analytical equations has been introduced; a major change to the way of calibration used before [2]. This can be applied to a large variety of lidar systems, and will be discussed for one specific lidar within the Met Office Volcanic Ash (VA) network [3, 4] in the following. Using this new approach, the linear volume depolarisation ratio $\delta \mathrm{V}$ can be expressed as:

$$
\delta V=\frac{\delta^{*}\left(G_{T}+H_{T}\right)-\left(G_{R}+H_{R}\right)}{\left(G_{R}-H_{R}\right)-\delta^{*}\left(G_{T}-H_{T}\right)}(1)
$$

The parameters $\mathrm{G}$ and $\mathrm{H}$ account for effects without and with atmospheric polarisation in the reflected $(R)$ and transmitted $(T)$ channel (explicit definitions can be found in [1]), respectively. The measured range dependent signal ratio is here defined as:

$$
\delta V=\frac{P_{R}(r)}{\eta P_{T}(r)}
$$

The calibration factor $\eta$ can be determined from the gain ratio $\eta^{*}$, which we get with a $\Delta 90$ calibration, and the theoretical correction $\mathrm{K}$ of the gain ratio.

$\eta=\frac{1}{K} \eta^{*}=\frac{1 P_{R}}{K P_{T}}( \pm 45)$

The correction $\mathrm{K}$ can be retrieved from an analytical expression (see page 4203, 2016 [1]). Combining equation (1) -(3) we get:

$\delta V=\frac{\frac{P_{T}( \pm 45) P_{R}}{P_{R}( \pm 45) P_{T}} K\left(G_{T}+H_{T}\right)-\left(G_{R}+H_{R}\right)}{\left(G_{R}-H_{R}\right)-\frac{P_{T}( \pm 45) P_{R}}{P_{R}( \pm 45) P_{T}} K\left(G_{T}-H_{T}\right)}$

\section{METHODOLOGY}

In order to calculate the $\mathrm{G}_{\mathrm{R}}, \mathrm{G}_{\mathrm{T}}, \mathrm{H}_{\mathrm{R}}, \mathrm{H}_{\mathrm{T}}$ we need to describe the optical set up of the lidar using the Müller-Stokes formalism. The multiplication of Müller matrices is in direction of the propagation of light. In essence our lidar can be represented by the set up as shown in Figure 1.

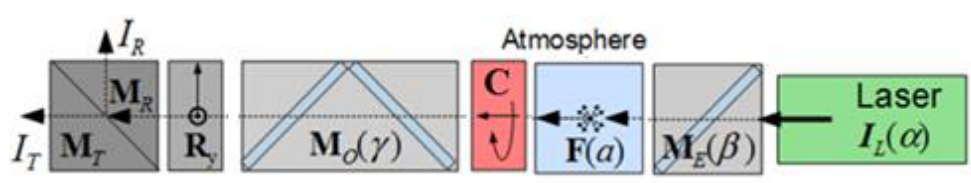

Figure 1: Müller-Stokes schematic of a twochannel, polarisation-sensitive lidar setup (adapted from[1].). Müller matrix block elements are indicated by $\boldsymbol{M}$ and the calibrator $\boldsymbol{C}$ is a rotator in our set-up. The elements are as follows IL: laser Stokes vector, ME: emitter optics; $\boldsymbol{F}$ : atmospheric backscatter matrix with polarisation parameter $a ; \mathrm{MO}$ : receiver 
optics; $\boldsymbol{R} y$ : rotation matrix for the $0^{\circ}(y=+1)$ and $90^{\circ} \quad(y=-1)$ detection setup (see text); $M T, R$ : transmitted and reflected part of the polarising beam splitter; IT,R: transmitted and reflected detection signals. Angles $\alpha, \beta$, and $\gamma$ are rotations around the optical axis.

\section{Application and calculation of the $G, H$, $K$ parameters with an example}

We will now describe how to obtain the needed parameters on the example of the datasheets provided for the Camborne lidar of the VAnetwork [3, 4]. The same approach can be applied to other lidar systems.

The correction parameters $\mathrm{G}, \mathrm{H}$ and $\mathrm{K}$ can be easily calculated using a Python code developed by V. Freudenthaler. The latest official version of the open source code is available from https://bitbucket.org/iannis b/atmospheric lidar ghk. The code works with Python 2.x and 3.x. The code reads the lidar system parameters from an input file (as downloaded from bitbucket and edited for a specific lidar). Therefore, we will use the same nomenclature as in the input parameter file for easy application to future studies. The input parameters $X$ are indicated in italic, their uncertainties as $d X$, and the number of steps in the error calculation as $n X$. In order to adapt the optical input parameters for our own lidar we need the optical performance values for each element. We define the plane of linear polarisation of the laser as the reference plane of our optical system.

\subsection{Polarizing beam splitter and cleaning polarizing sheet filters}

We start from the left in figure 1 with the PBS transmission $T_{S}$ and $T_{P}$ in the planes perpendicular $(S)$ and parallel $(P)$ to the incidence plane, respectively, which are given by the manufacturer for $355 \mathrm{~nm}$ as:
$T P, \quad d T P, n T P=0.9837, \quad 0.01,1$
$T S, \quad d T S, n T S=0.00035, \quad 0.0000035,1$

The retardance RetT is assumed to be $0^{\circ}$.
RetT, $d \operatorname{Ret} T, n \operatorname{Ret} T \quad=0.0,0 ., \quad 0$

All values of the reflected path of the PBS, i.e. $R P$ and $R S$ and their uncertainties, are directly calculated from the transmission values: $R P=1$ $-T P$ and $R S=1-T S$.

We use high quality polarisation filters behind the transmitted and reflected path of the PBS, to minimize the cross talk. Their extinction ratio ERAT and ERaR are given by the manufacturer:

ERaT, dERaT, nERaT $=0.00014,0.00002,1$
ERaR, dERaR, nERaR $=0.00014,0.00002,1$

The Rotation RotaT of the pol.-filter behind the transmitted path is $0^{\circ}+/-2^{\circ}$ and RotaR behind the reflected path is $90^{\circ}+/-2^{\circ}$ with respect to the incidence plane of the PBS.

RotaT, $d \operatorname{RotaT}, n \operatorname{RotaT}=0 ., \quad 2 ., \quad 1$

RotaR, dRotaR, $n$ RotaR $=90 ., \quad 2 ., \quad 1$

\subsection{Rotation matrix parameter}

As the laser polarisation rotation Rootle $=0^{\circ}$ and the parallel laser polarisation is detected in the reflected channel, the polarising beam splitter orientation parameter $y=-1$. This leads to the rotation matrix $\mathbf{R y}$ :

$$
R(y)=\begin{array}{cccc}
1 & 0 & 0 & 0 \\
0 & -1 & 0 & 0 \\
0 & 0 & -1 & 0 \\
0 & 0 & 0 & 1
\end{array}
$$

\subsection{Receiver optics including telescope}

The diattenuation parameter is defined as $D=(\mathrm{I}$ - I ) / ( I + I ) (see [1] p. 4190, Eq.(15)) of the $355 / 387 \mathrm{~nm}$ beamsplitter as given by the manufacturer (Raymetrics, pers. Communication):

$\mathrm{DiO}, \mathrm{dDiO}, \mathrm{nDiO}=0.0075567,0.0008,1$ $\mathrm{TiO}=0.9925$ 
Ret $O, d \operatorname{Ret} O, n \operatorname{Ret} O=0 ., \quad 0 ., \quad 0$

RotO, $d \operatorname{Rot} O, n \operatorname{RotO}=90 ., \quad 0.5, \quad 0$

\subsection{Calibrator}

We use a mechanical rotator before the receiver optics, which is described by the calibrator and location parameters:

LocC $=3 ;$ TypeC $=1$

\subsection{Emitter optics}

For two rotated retarding linear diattenuators (in our case two steering mirrors, one before and one after the beam expander) at $0^{\circ}$ and $90^{\circ}$ with respect to incident plane, we get for the combined diattenuation $(\mathrm{DiE})$, transmittance $(\mathrm{TiE})$ and retardance (RetE) (adapted from [1], eq. 10.10.10):

$$
\begin{gathered}
D i E=\frac{D i 3-D i 4}{1-D i 3 D i 4} ; \\
T i E=T i 1 T i 2(1-D i 1 D i 2) ; \\
\text { RetE }=D i 3-D i 4
\end{gathered}
$$

Both highly reflective mirrors were custom designed (Laseroptik $\mathrm{GmbH}$ ) so that the phase shift is zero at $355 \mathrm{~nm}$ and the reflection (here referred to as $T i E>0.95)$. The polarisation state, when the laser beam hits the incident plane, is considered as s-polarisation. Because the mirrors are cross aligned, their effects for $D i E$ and RetE cancel each other $(D i E=\operatorname{Ret} E=0)$. The intensity transmittance is $\mathrm{Tmax} \sim 0.97$ for s-polarisation and Tmin $\sim 0.93$ for p-pol. (see Figure $2 \& 3$ ).

This leads to the following parameters:

$\begin{array}{llll}\text { DiE, dDiE, nDiE } & =0.0, & 0.0, & 0 \\ \text { TiE } & =0.9025 & \\ \text { RetE, dRetE, nRetE } & =0 ., & 0 ., & 0 \\ \text { RotE, dRotE, nRotE } & =0 ., & 0.0, & 0\end{array}$

RotE (rotation of optical element in degrees) only matters if RetE and $D i E$ do not cancel out (e.g. if angle between mirrors is $\neq 90^{\circ}$ ).

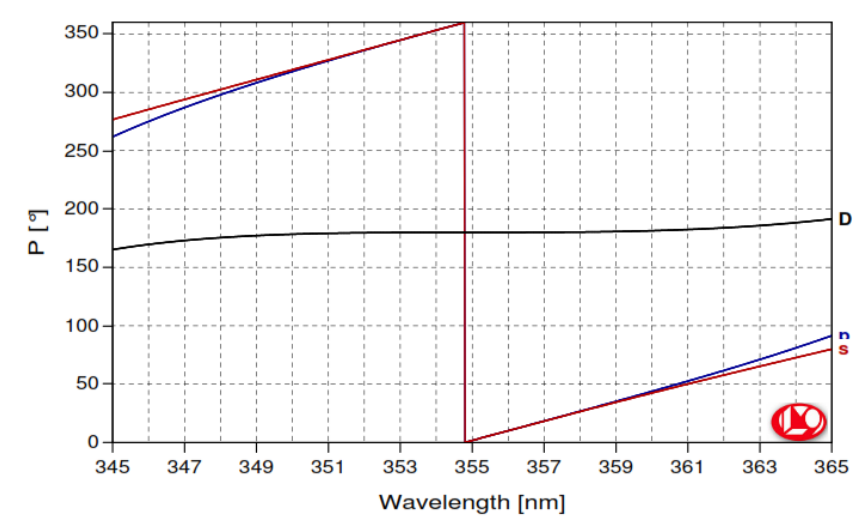

B-12046: R>97\% 355nm/s HT532nm/s HT1064nm/p 45phase

Figure 2: Retardation of the two mirrors in the emitting optics.

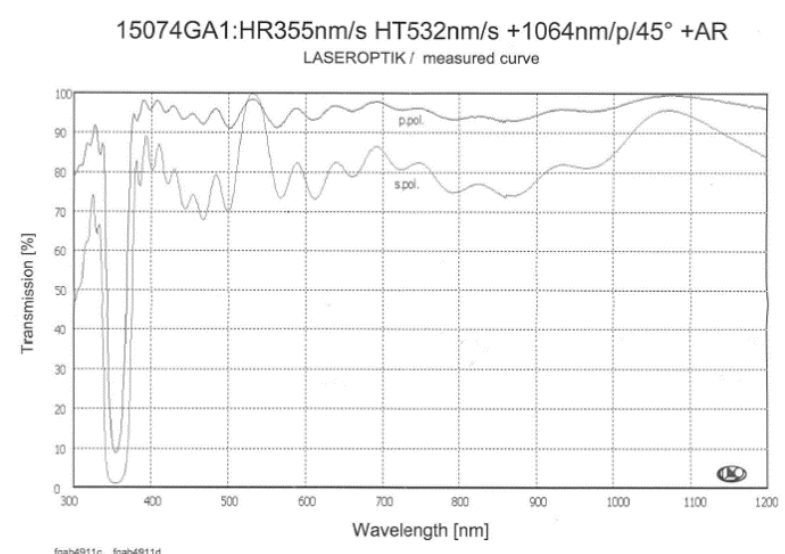

Figure 3:Transmission of the two mirrors in the emitting path

\section{Laser}

Specifications for the DOLP or the rotation of laser polarisation $(\operatorname{Rot} L)$ in degrees have not been provided or measured. The polarisation purity of the laser was given to $99.7 \%$ by the manufacturer; so we assume as best case:

$D O L P, d D O L P, n D O L P=1.0,0.00$

RotL, dRotL, $n$ RotL $\quad=0.0,0.0 \quad 1$

The effects of other RotL and DOLP on $\delta V$ are evaluated further down in the results section and in an experimental study (see contribution to ILRC29 by M. Osborne). 


\section{RESULTS for $G, H$ and $K$}

We can now add the values from above in the input file for the Python script ver. 0.96. This results in:

GR, GT, HR, HT, K (0.004), K (0.2), K (0.45)

$$
1.95277, \quad 2.01411, \quad 1.94690, \quad-2.00807 \text {, }
$$
$0.984654,0.984654,0.984654$

$\mathrm{K}$ is evaluated for different $\delta V$ in the calibration measurements.

We further asses the effect of the laser's DOLP and $\operatorname{Rot} L$ on $\delta V$ shown in figure 4. We find a $\delta V$ of 0.0298 for the best case scenario $(\operatorname{Rot} L=0, \quad D O L P=1)$, which also is in agreement with the former way of calibration [2]. We find that $\delta \mathrm{V}$ ranges between 0.0181 0.0298 for RotL between $4{ }^{\circ}-0^{\circ}$ and DOLP between $0.99-1$, respectively and $\delta \mathrm{V}=0.0283$ assuming $D O L P=0.997$, $\operatorname{Rot} L=0 \circ$, as given by the manufacturer. This means an added uncertainty of up to -0.0102 to +0.0015 of the $\delta V$ in this case.

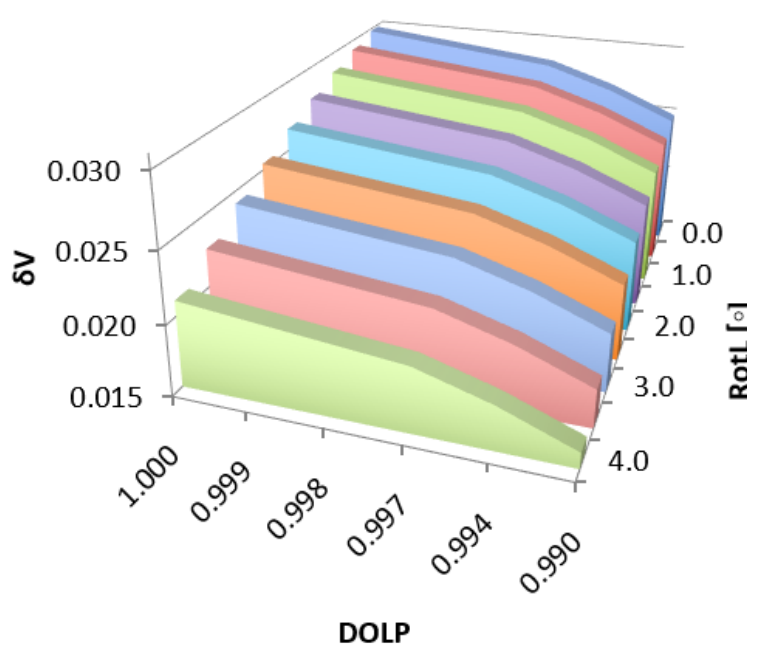

Figure $4 \delta \mathrm{V}$ depending on variation of DOLP and RolL.

\section{CONCLUSION}

We show how to practically apply the calibration method as described in [1]. We find, that additional assessment of values such as the rotation angle of the plane of polarisation of the Laser RotL as well as the DOLP need to be measured or additional uncertainty needs to be added to the calculated $\delta \mathrm{V}$.

\section{ACKNOWLEDGEMENTS}

The VA Lidar network is funded by UK Civil Aviation Authority and Department for Transport.

We thank T.Allott for managing the network and all the engineers for their hard work.

\section{REFERENCES}

[1] Freudenthaler, V., Atmos. Meas. Tech., 9, 4181-4255. (2016).

[2] V. Freudenthaler et al. Tellus, Ser. B Chem. Phys. Meteorol. 61(1) 165-179 (2009)

[3] M. Osborne et al. Atmos. Chem. Phys. 19(6) 3557-3578. (2019).

[4] Adam, $M$ et al., Proceedings of the 28th International Laser Radar Conference. (2017). 